\title{
Fundamentals and Limits of the Inclusive Culture and Inclusive Practise
}

\author{
Christian Wevelsiep \\ Department of Sociology, University of Flensburg, Flensburg, Germany
}

\section{Email address:}

familiewevelsiep@t-online.de

\section{To cite this article:}

Christian Wevelsiep. Fundamentals and Limits of the Inclusive Culture and Inclusive Practise. Education Journal.

Vol. 8, No. 6, 2019, pp. 320-326. doi: 10.11648/j.edu.20190806.22

Received: June 28, 2019; Accepted: July 24, 2019; Published: November 25, 2019

\begin{abstract}
Inclusion is an ideal: Belonging to the educations system is not a question. The children with disabilities are all part of a group, of a class, a school and of a community. This idea is great, but it needs to be grounded. Before describing in concrete terms which possibilities open up inclusive practices, the negative must be taken in account. The negative is intrusive: for the claim - to "integrate" everyone - can fail at certain moments. The idea of promoting and not "selecting" ends with a disturbing insight that affects a certain group: children with emotional and social developmental disorders. An interesting but difficult group, if we allow ourselves a few cliché-like exaggerations. A group that will be described in the following. The following article therefore covers a very wide range of objects. First, it aims to recall the foundations of inclusive theory; in this respect, it primarily aims at social-theoretical idealizations for which there is no exact equivalent in reality. First of all, abstract norms and values are at stake, ideals that always receive a resonance in pedagogical reality. But this resonance is not a measurable effect that can be exactly reproduced with the means of empirical social research. On the other hand it is a question of a group of people who may not be able to correspond to the described ideal: Children and adolescents with social and emotional developmental disorders. This group is trivially a factual component of a larger social group. But to what extent their integration and promotion is feasible within an inclusive framework would be questionable. Accordingly, the methodology must remain related to phenomenological perspectives. For it is not just a question of asking who may be a victim of exclusion, who is successfully integrated into a system and who is excluded. Rather, it is about the social-theoretical consequences of an irreversible tension: between a reality in which educational subjects do not correspond to the expectations, in which children are "sorted" and "classified" - and a theory that would have to draw conclusions from this situation. The aim of the following considerations would therefore be to start from the perspective of those subjects that we describe as inferior and marginalized, and to what extent observing the preconditions of this group should be a constitutive (and hitherto overlooked) component of the theory of inclusion.
\end{abstract}

Keywords: Inclusion, Power, History, Exclusion, Disability

\section{Introduction}

The look into the history of exclusion is a gloomy, almost ominous one. It is inevitable because we need realism in order to correctly assess the downsides of modernity and the effects of modern power. In this sense, one needs a critical theory and we also need the "altitude" of theoretical abstraction, which keeps false states before our eyes: exclusion, the falling out of integral references.

Exclusion, as we know, has an ambivalent counter-concept: Inclusion. One speaks of an inclusive culture and inclusive structures. What makes such an culture inclusive, if it is not reduced to the cool distance of the sociologist? Perhaps it is necessary to ask a different question and not to put inclusive culture as a means against experienced powerlessness, not to condemn one perspective and value the other. We may have to proceed differently and ask about the chances of an inclusive culture within a framework shaped by the mechanisms of exclusion. We must keep realism in mind. This also means that we do not have to change the whole thing, even if this seems to be the only way out for a postmodernism that is misleading. These problems are being negotiated elsewhere.

This is about creating proximity, not the big picture; it is 
about successful coexistence, not about last regulations and rules. Let us first think of the opposite of inclusion. What is the experience of exclusion like when it becomes "corporeal", when it appears as a hard causality that has to be overcome? There would be countless stories to tell, but the focus should be on those individuals who are hit particularly hard by exclusive mechanisms: Children and adolescents. If they are experienced immediately, mechanisms of exclusion prove to be long-lasting and memorable. They are condensed into biographies and life worlds that illustrate the difference between the integrable and the non-integrable. At the point at which the hardness and heaviness of the living world becomes perceptible, the pathos of inclusion is demanded: because only when one manages to integrate those individuals who have not had the easiest biographies behind them, who - metaphorically speaking - carry a heavy "backpack" and are often burdened - only then can the validity of inclusion prove itself.

So before describing in concrete terms which possibilities open up inclusive practices, the negative must be taken in account. The negative is intrusive: for the claim - to "integrate" everyone - can fail at certain moments. The idea of promoting and not "selecting" ends with a disturbing experience: that individuals take themselves out of the game, that they endanger themselves and others without regard for losses, and probably also without regard for inclusive ideals. The following is a brief description of what is meant here by the terms inclusive culture and inclusive practice (1). This is followed by reflections that take a phenomenological look at a specific group (2). How general statements about bonds and violations can be combined with the ideal of inclusive theory is shown last (3-4).

\section{The Meaning of "Inclusive Culture"}

In the feuilletons and the daily publications you will find reports of experiences which refer to the current state of inclusion. Not only the forms of work were redefined, but also the social references. Children and adolescents with learning and development disorders have not been sorted out for a long time, but assigned to the rule systems. Children with disabilities have the right to inclusive schooling, and educational institutions located above the special education system have the duty to admit all pupils without reservation, without a minimum qualification, without conditions. This is how things could be summarized in the narrowest sense, without regional differences, without naming any subtleties in school law or school organisation. The enforcement of a right - nothing else means inclusion for many - has its price. It is difficult to say how the individual groups will come to terms with the new circumstances. However, the mood is very clear when one summarizes individual contributions, critical comments, the formation of initiatives or the general pressure on the school authorities.

Inclusion is an ideal theory. In the ideal sky of an inclusive culture, there is simply no minimum that one must satisfy, no decisive criteria that one must fulfill in order to belong.
Belonging is the first criteria, one does not have (still in the semantics of theory) to fight for it [1]. The children with disabilities are all part of a group, a class, a school and of a community. Without taking a negative look at obstacles to development; no one is "sorted out" if they do not live up to expectations [2]. This idea is great, but it needs to be grounded. Then it will certainly not be abandoned, but tested in the concrete case and special conditions. From a technical point of view, this test affects a special group: children with emotional and social developmental disorders. An interesting but difficult group, if we allow ourselves a few cliché-like exaggerations. A group that will be described in the following in one case.

\section{Cannot Be Integrated? Children and Adolescents in Difficult Life Situations}

In the sphere of science there are various ways to demonstrate that inclusive practice has advantages in terms of equal opportunities. One could, for example, assume the opposite and try to show that the practice of segregation in special education institutions has a negative effect on the development of marginalized pupils [3]. Or one could concentrate on practice-oriented ways to improve and even optimize the integration of disadvantaged children and young people in education [4].

One alternative that will be taken in the following is a phenomenological description. To this end, it is not first asked about effects or scientifically verifiable interventions, nor is it assumed that a particular group always falls outside the grid of integration/inclusion. It is merely described (with implicit reference to pedagogical orientations in familiar living environments) under which aspects the group of emotionally/socially disturbed children and adolescents could be represented in everyday pedagogical life. These descriptions do not, of course, serve the purpose of overemphasizing their negative sides; they are neither to be expressed in stereotypes nor to be portrayed as pathological in their behaviour. The descriptions concentrate on what proves to be aggravating and what has to do with interpersonal suffering.

Alexa, 9 years old, comes from an Eastern European region that may be politically integrated, but is still an untapped space. The family didn't want to stay in a world that doesn't hold any promises. They, father, mother, four children, were drawn to a foreign land, to that part of Europe that offers a better life. New worlds were opened up there, but the problems did not diminish. The divorce was followed by new life partners, new insecure relationships. The child's mother's poverty became a problem, mainly because it was coupled with difficulties, the educational systems being highly problematic. Poverty and violent upbringing went hand in hand. The effects of marginalization and an educational climate that must be described as illegitimate interacted. In such a case, things are not turning for the better, but can take on a scale that is difficult to bear and that 
requires social work.

It is a "case" that will not surprise anyone who deals with it more intensively. But what does it mean for the question of the integratability of a particular person? Alexa, which is the subject of this paper in an exemplary manner, showed developmental delays and behavioural abnormalities in view of the conditions described above. Phenomenologically described (and close to the authors 'own experience): Alexa takes part in school events, but more and more often she takes a "break". This means that she flees from an unpleasant situation in which she is challenged. She avoids the communication after the conflict, she avoids her own positioning or a statement. Strife and violence are nothing conspicuous or special in schools, they can be dealt with. But the nature of a psychological impairment in the sense of a serious developmental disorder can be classified differently. What is normally "mastered" in other cases with mediation and communication, empathy and rule-consciousness remains a problem here. Conflicts are not created out of the world by good will. Rather, the participants fail because of the extent and intensity of the disturbance patterns and the structural violence [5].

Intensity: in this case this means patterns of behaviour that show depth, persistence, duration and tendencies towards danger. It is not just a question, as many textbooks describe, of a disturbance of the usual course of events.

Teaching has priority, but interruptions occur naturally. Some examples: Hans kicks his neighbours. Lea cries because she is afraid of something. Phillip emerges through clowns and provocations. The teacher's strategic and communicative skills are required in this case.[6] What about Alexa?

The phenomena described above may occur, but they are broken by more profound behaviors: Inexplicable outbursts with emotional verve. Distance through insults and at the same time the creation of closeness, also physical closeness, which must have a mysterious effect on those involved. Certainly also behavioural disorders that resemble border crossings: biting or spitting. Things that can be called pathological, but make sense in the horizon of a single child's life. In this case, it can be summed up, it is a matter of the phenomenon of a disorder of attachment. Not, as one would suspect, the marginalization and poverty of the family, not the miserable material circumstances are at the center. Rather, the aggravation of an already strained situation through massively disturbed relationships. Up to this point it would be understandable to follow the reflex of a professional distancing - is this not a case for psychological experts? Shouldn't pedagogical questions be reinterpreted into clinical, psychopathological questions? But let us stick to the case described above. A binding disorder such as the one indicated can have many causes.[7] It can be seen in patterns and tendencies that sometimes appear inconspicuous and can be corrected, but it can be unfolded with all force elsewhere. In these cases, experts speak of children with safe and insecure bonds. What is immediately obvious and cannot be doubted is that the development chances and risks of an infant are determined by the quality of early bonds. The entire development, the discovering social and play behaviour, social relationships and emotional needs are steered in tracks that are familiar to us: when children act and feel self-confident and bonded, when they act in the horizon of an emotionally secure relationship because there are just enough reference persons available. One speaks of children who are safely tied and who are better able to learn than children who are not safely tied.

\section{Between Violence and Understanding}

The following will describe to what extent a specific life situation, which we describe and evaluate as violent, poses a particular challenge to pedagogical practice. First it is merely pointed out that this violence (which does not have to be exclusively extreme or endangering for the welfare of the child) cannot simply be compensated or treated, but that this violence is a constitutive component of this very life.

The intensity of the disturbance patterns associated with the experience of insecure attachment must be questioned. It would also be necessary to ask what exactly safe attachment means in each case - there is no question that the motherchild dyad has priority, but further aspects need to be addressed: how important are other reference persons at what age or which positive experiences need to be added in order to "catch" the development? [8]

Let us stick here to the elementary challenges that a child with an insecure bond presents us with. The fact that it shows insecurity in the sense mentioned will not be doubted by the people around it. In the case described, hints are sufficient. A relationship between a parent and a child that appears to be "disturbed" is complex, dramatic and therefore dependent on detailed descriptions. Every short-winded analysis is forbidden - a look at the most terrible exceptional situations with child endangerment as well as a superficial comparison: the fact that people act incorrectly and imperfectly, hurtingly and inappropriately in relationships does not help as a general insight in this case. One may not necessarily understand the depth of a disorder of attachment if one looks exclusively at the moment of violence - which of course does not release one from the obligation to prevent endangerment by violence with all necessary means. But the essence of a bond disorder is opaque and difficult to grasp: because something is missing. An inkling of this is enough to realize that language fails at that point - what answers should it provide? The language that we and especially the children concerned have at our disposal is not the language that is generally spoken. If something is missing in a interpersonal or inter-existential relation, one can wish for it, demand it or simply complain about it. Language helps us in situations that are transparent and insightful, whose conditions we see through. Language then makes causal connections that guide us. But when inscrutability, insecurity, fragility determine life, language can fail, or at least it cannot produce the desired clarity [9]. This general context concerns vulnerable children in particular. Contexts can be guessed at, but not 
finally be understood. Problems that exist in the adult world can be "discussed". But the fact that this respective world of the others is interspersed with suffering means that the linguistic horizons and possibilities of expression are missing in the child's consciousness. Cliché-like ideas support what has been said: violence never comes down from heaven, it has a history. In the adult world there are experiences of violence and ruptures that do not heal. In the case of Alexas' mother, it was experiences of rigid and heartless upbringing that go back a long way - as well as historical humiliation and coldness, which had a direct effect on the environment. This violence is not simply passed on, even if there are milieus that supposedly "inherit" their bad sides.

It is rather the case that something in the background guides the superficial motifs, that the unconscious is expressed elsewhere. In concrete terms, in this case it would be permanent overstraining, emotional coldness, educational errors, which might be accompanied by rigid sanctioning. Unfortunately, as in this case, there is also a lot of violence at play, but perhaps not the violence that we would immediately classify as a threat to the welfare of the child.

Fatally, one could say, concrete, physical violence is the simplest form of violence. Terrible for the children affected, but somehow tangible and manageable in their life form and environment. Rights are violated and, if this becomes known, constitutional and psychological measures can be taken. This remains difficult enough for all who are concerned. But the other form of violence is more tragic, so to speak: there is often a mixture of disregard, overtaxing, overwhelming, and where perhaps loving understanding should set the tone, cold prevails. Where reliability and security should exist, rejection, disinterest or excessive severity rule. What is added was indicated above: exhaustion that arises from the dynamics of exclusion and can hardly be slowed down at a single point [10]. It is now the question of what value we attribute to the case described. The introspection into a world like the one described must not be unduly claimed, it can hardly be used for a larger theory. It serves here as a preliminary understanding of what we can and must grasp under an emotional developmental disorder in the broadest sense. It is an approximate, doubtful understanding, not a total view of an event in which we are never involved. Even the greatest possible empathy only serves the purpose of sounding out the conditions that cause and advance developmental disorders. One can at least guess up to this point that the conventional means fail, that an additional support lesson, a special learning support or a "support plan" in this case miss the point. Special attention needs to be paid to situations in life which act as a breeding ground for developmental disorders, far beyond the material and the effective.

\section{Two Ways of Integrating a Group with Special Needs}

Social-emotional conspicuities are of course only a possible appearance, an aspect of the much larger topic of life under impairment. But the step towards a more general theory of the disorder is not so big: in this context one speaks of a difficult learning and living situation. An aggravated situation - this hits the crucial point. It is the difficult situation of a child who has to cope with the most difficult circumstances. But this situation is also a difficult overall situation, for the family, for all individuals who come into contact with the matter. There are two ways of integrating the mentioned problems into the context of inclusive practice and theory.

First. Inclusion can be understood as a regulative principle that "works" in the vast majority of cases, but which always meets a limit, a limit at which conventional means fail. In this case one would speak of such a strong, so manifest disturbance that the question of inclusion does not arise at all. The behavioural problems are like states of exception in which neither reasonable practice nor our pedagogical resources are sufficient. The extent, depth, persistence of the disturbance go beyond the scope of what we are talking about in terms of inclusive practice. This interpretation is comprehensible. One is subject to a logical reference to first get the most urgent problems into view and to put all higher goals at the back. Nevertheless, there is a catch. It is assumed that one can precisely assess the severity of emotional developmental disorders. Of course, distinctions can be made: a child with hyperactive behaviour can be integrated if the conditions are designed accordingly; a child with cold aggressions, which reach a "pathological" level, needs more intensive support and short-term "special education" (which is not equivalent to a spatial separation). The crucial point, of course, is that this distinction is subject to different perspectives of judgement, horizons of experience and evaluations. A behavioural disorder is always an attribution of authority to an inferior subject. How should a doctor, a young teacher, a dean of studies, a specialist chairman of an education commission, a psychotherapist, a forest pedagogue or a social worker - in order not to leave out any perspective recognize or set an objective standard? So many perspectives, which flow into the evaluations, so many expectations of an environment to judge someone as acceptable, integrable or even unbearable!

This boundary can be shifted in two directions in one's own interest: to the side of the "system", which has priority through its formal rules and norms. Any violation of the rules would then be a reason for exclusion from the system. But also on the side of the subject, who in this case needs priority and should exercise his rights. It would be easy if one could argue in terms of human rights here: because if the rights of others are violated, i.e. if children act to an extent that endangers others, the legal consequences can be drawn. But even in this case things are never as clear as one might wish. When are border violations permanently dangerous? When may we "train" children in a legitimate way to protect others, when does the logic of the disciplinary state apply, when does the logic of human rights apply?

Two extreme forms show that it always depends on our evaluations, which cannot lay claim to objectivity. One 
example: In the USA, violence, drug problems, crime have always been topics in schools as well as existing discrimination or ethnic segregation. In order to get the problems under control, measures were tested, school laws were passed and rejected again, initiatives were donated. Such initiatives were and are recognizable in the linguistic call for inclusion: No child left behind! But also in the form of strict control and discipline, when one thinks of so-called zero tolerance programs. The basic idea is to be rigorous and adaptable, to avoid adopting a laissez-faire attitude unconditionally. No deviation from the rules of an institution should be allowed from now on, which in this understanding of course also means: no possession of weapons or drugs, no crime, no violence. Expectations that should not be discussed or negotiated any further. The difficulty arises with regard to the measures: it was made easier for the authorities to remove the "accused" from the education system as a whole. The reason: these groups were untrainable, non-integratable, incorrigible. The labels were clearly brought into the public eye: these were situations which were not only difficult but also impossible to solve, a diagnosis from the field of religious meaning - one would have to work with unredeemable children [11]. In this case, the trap axe of exclusion falls quickly and it is not surprising that this was also accompanied by ethno-political constructions. The more general insight is that the judgement of an instance is massively influenced when the framework conditions are changed. For example, when a basic education law becomes a minimum qualification for general belonging in a system. In extreme cases, the right to education is tied far too closely to the respective good behaviour.

But we also have to look closely from the other side. Here, too, the diagnosis is difficult, but the voices that warn of the rampant violence in schools must certainly not be overheard. Mood pictures can easily be exaggerated. The diffuse fear that violence will become more extreme and manners rougher can always be exploited. But of course it must not go so far as to expose the educational authority to constant violence, not only from pupils, but from a whole social environment in the broadest sense. The indifferent ignoring of a pupil with aggressive portions would be the wrong consequence in this case and it is an open question under which conditions the respective school authorities in Germany or France, in Western Europe or in the USA or elsewhere react to comparable threats [12].

If we consider these sides, then it would be understandable to remove the manifestations of violence from an inclusive system. Inclusion is therefore a yardstick that offers us orientation in peaceful everyday life and sovereignly disregards the state of emergency. Developmental disturbances that are accompanied by violence and extraordinary manners would therefore have to be placed in a different area of order. This consequence is in certain respects compelling and it is an expression of a practice. For example, when children are taken out of their accustomed environment for a longer period of time and psychiatrically cared for. But is this mandatory? Or can this consequence be avoided in another categorical reference?

Secondly. There is another way, which is not easy to convey and leads to unsafe terrain. One can understand the inclusive practice as a commandment that exists in a certain sense without further conditions. There have to be no exceptions. In other words, the severity of a disorder, the extent of a disability or even the intolerability of a behaviour must not be the condition that dictates the validity of a principle. Inclusion would basically be misunderstood: it would only come into force if the practice was comfortable and the efforts were small. This is polemic and "cheap" - and one can well imagine the outcry: the lamentation of the professionals who are exposed to extreme stress, who have experienced the culture of inclusion as a permanent overload. These voices must not be overheard, they must be taken seriously - but the first thing here is to defend a principle. The culture of inclusion does not end at the point where one recognizes an extreme lack and inadequacy in the social sphere. The opposite conclusion, of course, is not reached either: that we should enforce an inclusive practice under all circumstances and give preference to an ideal over reality. Rather, it is questionable how we can deepen the culture of inclusion and advance its practice without leaving individual groups on the margins. At first, this is an idealistic idea that is only tested and played through in theory here. But it is important to emphasize that an inclusive practice takes place on an idealistic ground and that it would be wrong to make corrections to the ideal of what is feasible.

\section{A Conclusion: Inclusion in a Contradictory World}

A philosophical digression can perhaps confirm this: Jürgen Habermas' discourse theory has rightly assumed an important position in contemporary philosophy [13], but it is also to be understood as a contribution to social understanding. When we enter into an argument, we are subject to certain conditions. These conditions are negative: we do not exclude anyone from a discourse from the outset, we assume reciprocally equal rights. We do not deal with each other in a certain way: violently, despisingly, hostilely. Nothing speaks against polemical exercises and a fierce argument, but these things only work if these requirements remain fulfilled. One can call it the non-rule discourse, in which the non-violent power of the better argument prevails, in which something ideal emerges. To put it more simply, it is about granting everyone the same rights and the same voice. Applied to the culture of inclusion, this means that no group should be treated differently and excluded as deviant because of its lack of qualifications or its individual possibilities. That contradicts now first of all what different specialists express very clearly: that evenly those group of the emotionally and socially retarded children can experience 
an inclusive group as extreme load. As contradictory as things may seem here: this step - to bring a special resilience and reasonableness into play - would only be the next. If we understand inclusion only as a half-hearted principle and make its validity dependent on successful interactions and encounters, the original idea would have failed. In fact, the opposite way makes sense and it also has a philosophical dimension of depth. Our lives include aspects that we must call fragility and finiteness, whether we like it or not. Philosophically, the possibility of exposure and powerlessness is an insight of the highest value. But it can also be formulated more simply: we always enter life by presuming failure and suffering. Our understanding never succeeds perfectly, but is always inadequate - regardless of whether or not we base ourselves on an ideal practice. Even more fundamental than the insight into reasonable arguments is the thought of mutual concealment, incomprehension, doubt and failure. At first glance, this speaks against the outlined practice of ideal communication.

The feeling of being exposed may not be equally familiar to everyone, but the factual basic situation affects everyone. So we don't have to cut back on the communicative ethos, we have to keep the initial conditions in mind. Inclusion is therefore not "up for discussion" because it starts as an idea at the fundamental conditions of being human. Every human being is vulnerable and needs special protection. We are all in a basic situation characterized by want, need and suffering. What can be concluded from this, if at the same time we know from our experiences that some have a higher need than others? If we also know that others suffer worse and differently than ourselves? So what do we conclude from the simple fact that the threat of suffering affects everyone in principle, but that singular life is permeated by a variety of violent relationships? This difference in the conditions of life, physical-somatic, social and political, material and sensual, suggests the assumption that certain people are particularly vulnerable in certain situations. This thought ignites, so to speak, the impulse of inclusion. The right conclusion would not be the separation of a special, needy being from its peers, but the consideration of the situation in an inclusive framework.

If we start from these fundamental premises, then the perspective shifts to the relationship between the general and the particular. The general principles can be defined as follows: by virtue of inclusive practices, pedagogical attention is not linked to prior qualifications. There is no achievement, no better or worse, no good behaviour and no head note that allows access to an unavailable right to education. There are no inclusive conditions for some and special disciplinary rights for others. This also means that children with emotional and social injuries are part of an inclusive education system.

So the conclusion is: The existence of the endangered subjects can be denied, overplayed, overlooked. It can be isolated by ignoring the contexts or by no longer accepting political backgrounds. Then, in the end, only subjects would remain whose right to exist had to be fought for. Inclusive culture has been fighting against this for decades; it can therefore be understood as a culture that gives the other a voice. The philosophical culture that gives substance to the whole cannot be traced back to the phenomenon of difference alone. The recognition of difference has its absolute justification, but it needs a deeper foundation. It should start with the fundamental question of the extent to which we must expose ourselves from birth to the help of others [14], but that our existence does not remain bound, as it were, to the one place that was eventually assigned to us. Neither in the geopolitical sense, neither in the face of worldwide migration movements, nor in the sense of an institutionalized normality. Inclusion requires spaces that can be left again, both physical and symbolic. In other words, it requires utopian potentials that emanate from the irritating other. These spaces are not fixed, they cannot guarantee any ultimate identity, even if one wishes that one could create equality in a social place as it were. The original solidarity in the utopian sense has no place: we do not "find" it in the welfare state, which distributes goods that at some point prove to be finite (which paradoxically explains its justification). Not in the classless society in which the solidarizing effect comes by itself. Not in one form of life, which is superior to others, whether socially romantic or selfish. We find it rather in the mysterious sense that it is the foreignness of life forms that we can acknowledge or misjudge [15]. This inclusive community is ahead of instrumental thinking because it enables friendship and closeness while respecting the limitation of the strangeness. This is not tolerance at a higher level, but a repeated crossing of boundaries that accepts the risk of conflict.

\section{References}

[1] Anne Waldschmidt: Rethinking disability. Cultural Studies Perspectives of Disability Studies. In: Ders. (Ed.): Cultural Studies Perspectives of Disability Studies. Tagungsdokumentation, Kassel 2003, p. 15- 20; Prengel, A.: Equality, Heterogeneity and Hierarchy in Initial Lessons and Beyond. In: Hinz, Andreas/Geiling, Ute (Hg): Integration Pedagogy in Discourse - On the Way to Inclusive Pedagogy. Bad Heilbrunn 2005, p. 15-34.

[2] Wember, F. B.: Inclusion challenge: A preventive-oriented model of school learning and four central conditions of inclusive teaching development. In: Zeitschrift für Heilpädagogik (ZfH) 10, 2013, pp. 380-388; Werning, R.: Das sozial auffällige Kind. Wiesbaden 1990; Ders: Remarks on a didactics of joint teaching. In: ZfH 11, 1996, pp. 463-470; Ders. (1998): Constructivism. A suggestion for pedagogy? Ders. (1998): Constructivism. A suggestion for pedagogy? In: Pädagogik, Issue 7-8, S. 39-41; Ders: Inclusion between innovation and excessive demands. In: Zeitschrift für Heilpädagogik 8, 2010, pp. 284-292.

[3] Wocken, H.: Performance, intelligence and social status of pupils with learning disabilities. Comparative study at special schools in Hamburg. In: Zeitschrift für Heilpädagogik 51, 2000, p. 492-503; Wocken, H.: An impossible essay on the therapy of an ill institution, in Journal for inclusion Zeitschrift für Inklusion-online.net, 2, 2013. 
[4] Willmann, M.: Increasing the educational competences of teachers through special educational consultation. In: Journal for Curative Education 6, 2007, p. 214-221; Wimmer, M.: Decay of the general - return of the singular. Pedagogical professionalism and the value of knowledge. In: Combe, A./Helsper, W.(Ed.): Pedagogical Professionalism. Frankfurt a M. 1996, S. 404-447; Wolf, C./v. Dick, R.: If otherwise does not mean worse. The appreciation of diversity promotes the equivalence of the group. In: Heitmeyer, W. (Hg.): Deutsche Zustände. Folge 6. Frankfurt a. M. 2008, p. 137-154; Wustmann, C.: The new focus of resilience research. How children cope with life stress. In: Zeitschrift für Pädagogik 51, 2005, p. 192-208; Tröster, H.: Chances of early recognition of aggressive-dissocial behaviour problems in the transition from kindergarten to school. In: Zeitschrift für Heilpädagogik 9, 2011; pp. 337-345.

[5] Hollstein-Brinkmann, H.: Social work and general systems theory. Freiburg 1993.

[6] Störmer, N.: "You are disturbing". Challenging behaviour and its interpretation as a behavioural disorder. Berlin: Frank and Timme 2013

[7] Fegert, J. M.: Quality - in every respect. In: Frankfurter Allgemeine Zeitung of 17. 11. 2012, p. 8.

[8] Götz, B.: The experience that it's not as it seems. About the difficulties of treating disadvantaged young people with respect. In: Baur, W., Mack, W. \& Schroeder, J. (Ed.): Education from the bottom up. Growing up in difficult life situations - provocations for pedagogy. Bad Heilbrunn/Obb., 2004, pp. 173-205.

[9] Thomas Rentsch: The Constitution of Morality. Frankfurt am
Main: Suhrkamp 1999; Häußler, M.: Skepticism as an attitude of curative education. Reflections on the professional ethics of curative education. Bad Heilbrunn 2000.

[10] Böttger, A.: Violence and Biography. A qualitative analysis of reconstructed life stories of 100 young people. Baden Baden 1998.

[11] Amos, S. K.: Zero Tolerance at Public Schools in the USA American Syndrome or Symptom for a New Determination of Social Membership and Educational Relations? In: Zeitschrift für Pädagogik 52, 2006, Issue 5, S. 717-713; Ayers, W./Dohrn, B./Ayers, R. (Ed.) (2001): Zero Tolerance. Resisting the Drive for Punishment in Our Schools. New York: New Press.

[12] B. Ahrbeck: Dealing with disability. Stuttgart: Kohlhammer 2011; Inclusion - a critique. Stuttgart: Kohlhammer 2014, pp. 14; Hinz, A.: Heterogeneity in school. Integration intercultural education - coeducation. Hamburg 1993; Ders.: From Integration to Inclusion. In: Journal for Curative Education, 9, 2002, pp. 354-361; Booth, T./ Ainscow, M.: Index for inclusion. Developing learning and participation in school. Bristol: Centre for studies on Inclusive Education 2002.

[13] Jürgen Habermas: Facticity and Validity - Contributions to the Discourse Theory of Law and the Democratic Rule of Law. Frankfurt am Main Suhrkamp 1992.

[14] Burkhard Liebsch: Exposed to each other - the other and the social. Elements of a topology of living together. 2 Vol., Freiburg/Mun ich: Karl Alber 2018.

[15] Christian Wevelsiep. Inclusion - about a fulfilling figure in common life. Duisburg: Athena 2019. 